\title{
Particle Transport Measurements in the LHD Stochastic Magnetic Boundary Plasma using Mach Probes and Ion Sensitive Probe
}

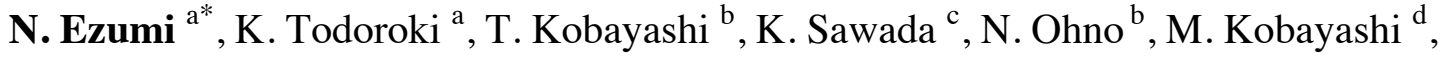 \\ S. Masuzaki ${ }^{\mathrm{d}}$ and Y. Feng ${ }^{\mathrm{e}}$ \\ ${ }^{a}$ Nagano National College of Technology, 716 Tokuma, Nagano 381-8550, Japan \\ ${ }^{b}$ Nagoya University, Nagoya 464-8603, Japan \\ ${ }^{c}$ Shinshu University, Nagano 380-8553, Japan \\ ${ }^{d}$ National Institute for Fusion Science, Toki 509-5292, Japan \\ ${ }^{e}$ Max-Planck-Institut fuer Plasmaphysik, D-17491 Greifswald, Germany
}

\begin{abstract}
Spatial profiles of the plasma flow, electron temperature $\left(T_{\mathrm{e}}\right)$ and ion temperature $\left(T_{\mathrm{i}}\right)$ in the stochastic magnetic boundary layer of Large Helical Device (LHD) has been studied by simultaneous measurements using a movable multiple functions probe, which consists of Mach probes and an ion sensitive probe. The tendency of the measured spatial profiles of $T_{\mathrm{e}}$ and $T_{\mathrm{i}}$ is similar to the three-dimensional simulation. The results of ion saturation current $\left(I_{\text {sat }}\right)$ measurement of the upstream and downstream probes indicate that the plasma flow direction is reversed in the stochastic magnetic boundary layer. $I_{\text {sat }}$ observations obtained deep inside of the boundary layer contradict the simulation result, even though the existence of flow reversal in the LHD stochastic magnetic boundary layer was qualitatively confirmed.
\end{abstract}

PACS: 52.25.-b, 52.30.-q, 52.70.Ds, 52.70.Nc

JNM Keywords: Experimental Techniques, Monitoring Methods, Plasma Properties PSI-19 Keywords: Edge plasma, LHD, Particle flux, Plasma flow, Probes

*Corresponding Author Address: 716 Tokuma, Nagano 381-8550, Japan

*Corresponding Author e-mail: ezumi@ec.nagano-nct.ac.jp

Presenting Author: Naomichi Ezumi, Presenting Author e-mail: ezumi@ec.nagano-nct.ac.jp 


\section{Introduction}

Particle transport is an essential and general issue and of particular importance for the proper functioning of the edge and the divertor plasmas. Both plasma flow and ion temperature $\left(T_{\mathrm{i}}\right)$ are the key parameters for characterizing the transport in the boundary plasmas [1]. The stochastic magnetic boundary field line structure has attracted attention from the viewpoint of the Edge Localized Mode (ELM) control in some tokamaks. Though the stochastic magnetic boundary layer exists intrinsically in the scrape-off layer on the ergodic divertor configuration of tokamaks and heliotron-type devices, plasma flow properties and ion dynamics in the layer have not been understood well.

Numerical simulations regarding the edge transport in the Large Helical Device (LHD) boundary plasmas have been done by the three-dimensional plasma and neutral transport code, EMC3-EIRENE [2, 3]. The EMC3 code calculates a set of stationary standard fluid equations in a real 3D space of arbitrary magnetic field geometry using an advanced Monte-Carlo technique. Since the remnant island width in the stochastic magnetic boundary is of order of $\mathrm{cm}$ or even larger at the low field side which it is much larger than the ion Larmor radius, the plasma transport is expected to be affected by such the field line structure. The calculation result has predicted the change of the plasma flow direction in the LHD stochastic magnetic boundary $[4,5]$.

So far, the alternating direction of plasma flow has been observed by using a multiple function probe in the edge of the stochastic magnetic boundary plasma [6]. In this paper, we show experimental results of plasma flow and temperature measurements using the probe in the deep stochastic boundary of LHD.

\section{Experimental setup}

Figure 1 shows a schematic of the multiple functions probe head designed for measuring the plasma flow, $T_{\mathrm{i}}$, electron temperature $\left(T_{\mathrm{e}}\right)$ and electron density $\left(n_{\mathrm{e}}\right)$ in the LHD stochastic magnetic boundary. The probe head consists of six single Langmuir probes and an ion sensitive probe (ISP) [7]. Pairs of probes on opposite sides of the probe head work as Mach probes. The probe tips were arranged at 60-degree intervals on the periphery of the 
shielding Boron-Nitride (BN) tube, which is $10 \mathrm{~mm}$ in diameter, such as the Gundestrup probe [8]. The dimension of exposed area of each electrode is $1 \mathrm{~mm}$ in height and $1 \mathrm{~mm}$ in width. These electrodes are put $1 \mathrm{~mm}$ inside of the BN tube. Tungsten (W) rods were used for the probe electrodes. The ISP for $T_{\mathrm{i}}$ and $T_{\mathrm{e}}$ measurements is put on the top of the probe head.

The concept of $T_{\mathrm{i}}$ measurement using ISP is based on the difference of the Larmor radii between ions and electrons. Typical ISP consists of two cylindrical electrodes which are an ion collector (inner electrode, P) and an electron guard electrode (outer electrode, G). W rod and a molybdenum (Mo) tube were used for the inner and the outer electrodes of the ISP, respectively. The distance between the top surface of the ion collector and the upper end of $\mathrm{G}$ electrode is defined as $h$. Assuming the typical plasma parameters and magnetic field strength in the edge plasma in LHD, the $h$ was set on $1.0 \mathrm{~mm}$ according to the theory developed by Katsumata [7]. The ion energy analysis can be performed by using the current-voltage (I-V) characteristics on $\mathrm{P}$ electrode. $\mathrm{G}$ electrode works as an ordinary Langmuir probe while playing the role of the fence for preventing electrons flowing into the ion collector. Note that ISP measurements basically have the same experimental limitation on plasma parameters as an ordinary Langmuir probe ones.

The Mach probes and the ISP work simultaneously. The multiple functions probe was installed on the top of the reciprocating type fast scanning Langmuir probe system [9]. It was inserted from the bottom port of LHD. Using this system, the probe head can reach the LHD stochastic magnetic boundary. Notice the incident angle of the magnetic field line to each probe is different as shown in Fig.1 (b). The probes were numbered from \#1 to \#6. In spite of the angle changes during the movement of the probe head in LHD, the angle is almost constant (40 43 degree) in $X-Y$ plane in the measured stochastic magnetic boundary in this study. Using pairs of probes on opposite sides, the difference in the incident field line angle is compensated because of the absolute angle is the same for each probe. The bias voltage for each Langmuir probe was set at $-200 \mathrm{~V}$ in order to measure $I_{\text {sat }}$ continually during the movement. The reference potential for all probe circuit was the ground (vacuum vessel's) potential. The resistances for detecting of $I_{\text {sat }}$ were $100 \Omega$ for each Langmuir probe. For $T_{\mathrm{e}}$ and $T_{\mathrm{i}}$ measurements, the probe voltage (triangular wave, $-200 \mathrm{~V}$ to $200 \mathrm{~V}, 200 \mathrm{~Hz}$ ) for the ISP was 
applied from the bipolar power supply to each electrode. The resistances of $1 \mathrm{k} \Omega$ and $1 \Omega$ were used for current measurement of inner and outer electrodes of the ISP, respectively. The resistances are enough smaller than the sheath resistance. These voltage signals across the resistors were fed to a digitizer via isolation amplifiers. The resolution and the sampling rate of the digitizer were 14 bits and 1 MSamples/sec, respectively.

\section{Experimental results and discussion}

\subsection{Spatial Profiles of $T_{\mathrm{e}}$ and $T_{\mathrm{i}}$}

The measurements using the multiple functions probe in the stochastic magnetic boundary of LHD plasma were performed in hydrogen plasma with NBI heating in the operational configuration with the magnetic axis position $\left(R_{\max }\right)$ of $3.75 \mathrm{~m}$ and a toroidal magnetic field strength of $2.64 \mathrm{~T}$ (Shot No. 84279).

Figure 2 shows the spatial profiles of $T_{\mathrm{e}}$ (open circles) and $T_{\mathrm{i}}$ (closed circles) measured by the ISP during the discharge of the shot number 84279 , where $Z$ indicates the position of the probe head from the center of the core plasma. The temperatures increase from around $Z=-1.0 \mathrm{~m}$. The region corresponds to the stochastic magnetic boundary layer. The results are consistent with the profile of connection length $\left(L_{\mathrm{c}}\right)$. Furthermore, the tendency of the measured spatial profiles is similar to the 3D simulation. The ISP measurement clearly shows the spatial profiles of $T_{\mathrm{e}}$ and $T_{\mathrm{i}}$ in the stochastic magnetic boundary layer.

\subsection{Spatial Profiles of Particle Flux}

Figure 3 (a) - (c) show the change of $I_{\text {sat }}$ obtained by each Mach probe during the probe head movement in the stochastic magnetic boundary. The results obtained by probes located on the opposite angle in the probe head are compared in each figure. As shown in Fig.3 (b), $I_{\text {sat }}$ of the probe \#5 shows larger value than the probe \#2 in the probe position $(Z)$ between -0.96 and $-0.925 \mathrm{~m}$. It means that probe \#5 and \#2 are located on upstream and downstream for the plasma flow, respectively. On the other hand, $I_{\text {sat }}$ of the probe $\# 2$ becomes larger than that of the probe \#5 in the position between -0.925 and $-0.895 \mathrm{~m}$. It indicates that the relation between probe positions and plasma flow is changed around $Z=-0.925$ and 
$-0.895 \mathrm{~m}$. The results suggest, namely, flow alternations exist around both of the positions. This behavior is also seen in the other pairs of probes as shown in hatched region of Fig.3 (a) and (c). These results are consistent with the geometrical structure of the probe head as shown in Fig.2 (b).

Figure 3 (d) shows a calculated parallel particle flux using the three-dimensional plasma and neutral transport code, EMC3-EIRENE, under similar condition to the experimental one. The power entering the edge region through LCFS is assumed to be $2 \mathrm{MW}$, which is boundary condition for the energy transport. Plasma density at the LCFS was set on $4 \times 10^{19} \mathrm{~m}^{-3}$ as the boundary condition. The sign of the particle flux indicates the flow direction. The flux with a plus sign corresponds to the flow from the side of the probe \#1, \#2 and \#3. Alternations of plasma flow are clearly shown at around $Z=-0.925,-0.895$ and -0.820 $\mathrm{m}$. The result for $Z=-0.925$ and $-0.895 \mathrm{~m}$ is consistent with the observed profiles of $I_{\text {sat }}$. However, no flow reversal is observed at $-0.820 \mathrm{~m}$ in the experiment. In order to estimate quantitative flow velocity or Mach number, precise analysis about particle flux is needed. It is important for the analysis to evaluate each effective collecting area of probe current. The estimation of the effective area is affected by the incident angle of magnetic field lines to the probe. Furthermore, sheath thickness around the probe electrode also has an effect on the area. The incident angle is changed three-dimensionally about the probe position, not only in the $X$,

$Y$ direction but also $Z$. Because of the existence of a strong $Z$ component of magnetic field, time difference has occurred between probes for crossing the same field line during the probe movement. The difference had been revised in analysis processes. Further work is underway to clarify precise flow velocity through the geometrical analysis and the detail investigation of the sheath structure around each probe by means of particle in cell simulations.

\section{Conclusions}

In this study, spatial profiles of the plasma flow, $T_{\mathrm{i}}$ and $T_{\mathrm{e}}$ in the stochastic magnetic boundary layer of LHD have been studied by simultaneous measurements using a movable multiple functions probe. Capability of the ISP measurement in the complex magnetic field structure has been confirmed. The tendency of the measured spatial profiles of $T_{\mathrm{i}}$ and $T_{\mathrm{e}}$ is 
similar to the 3D simulation. The results of $I_{\text {sat }}$ measurement of the upstream and downstream probes indicate that the plasma flow direction is alternated in the stochastic magnetic boundary layer. At deep inside of the stochastic magnetic boundary layer, the observed results of $I_{\text {sat }}$ contradict the simulation result.

\section{Acknowledgments}

This work was supported by NIFS Collaborative Research Program (NIFS05KLPP009 and NIFS08KLPP314) . 


\section{References}

[1] N. Asakura and ITPA SOL and Divertor Topical Group, J. Nucl. Mater. 363-365, 41 (2007).

[2] Y. Feng, F. Sardei, J. Kisslinger, P. Grigull et al., Contrib. Plasma Phys. 4457 (2004).

[3] D. Reiter, M. Baelmans, P. Boerner, Fusion Sci. Technol. 47, 172 (2005) .

[4] M. Kobayashi, Y. Feng, S. Masuzaki, M. Shoji et al. J. Nucl. Mater. 363-365, 294 (2007).

[5] Y. Feng, M. Kobayashi, T. Morisaki, S. Masuzaki et al., Nucl. Fusion 48, 024012 (2008) .

[6] N. Ezumi, S. Masuzaki, N. Ohno, Y. Uesugi et al., J. Plasma Fusion Res. SERIES 8 (2009) 429.

[7] I. Katsumata, Contrib. Plasma Phys. 36S (1996) 73-80.

[8] J. Gunn, C. Boucher, P. Devynck, I. Duran et al., Phys.¥ Plasmas. 8, 1995 (2001).

[9] S. Masuzaki and A. Komori, Annual Report of National Institute for Fusion Science, April 1999-March 2000, 87 (2000). 
Figure captions

Figure 1: The structure of the multiple functions probe head designed for the measurement in the LHD divertor and stochastic magnetic boundary. (a) Side view, (b) Cross section in $X-Y$ plane as seen in (a). Solid lines indicate the angle of the magnetic field lines in the measured stochastic magnetic boundary for each probe [6].

Figure 2: Spatial Profiles of the $T_{\mathrm{i}}$ and $T_{\mathrm{e}}$ in the stochastic magnetic boundary along the path of the probe. Solid and dashed lines are the results of $3 \mathrm{D}$ simulation for $T_{\mathrm{i}}$ and $T_{\mathrm{e}}$, respectively.

Figure 3: Comparison of measured ion saturation currents with calculated particle flux using 3D simulation code. The numbers in (a) - (c) correspond to the probe numbers defined in Fig.1. Flow alternations are clearly identified around $Z=-0.925$ and $-0.895 \mathrm{~m}$. (d) parallel particle flux $\left(n V_{\text {prara }}\right)$ calculated by the three-dimensional plasma and neutral transport code, EMC3-EIRENE, under similar condition to the experimental one. 
Figure 1

(a)

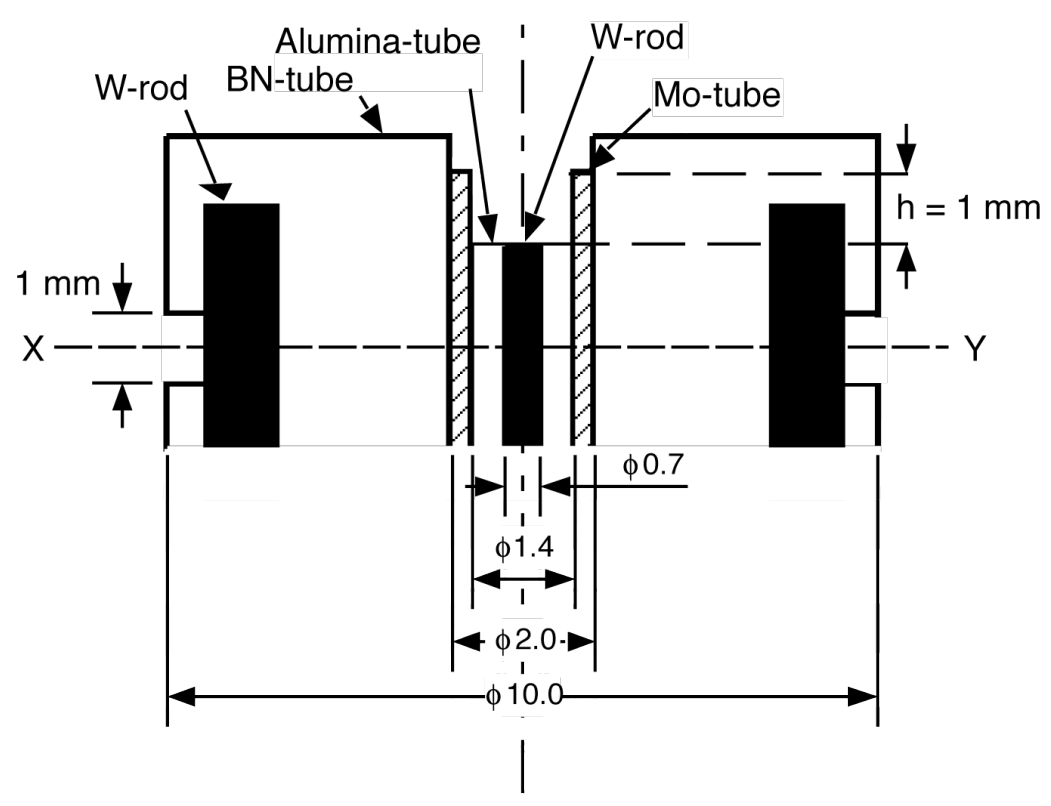

(b)

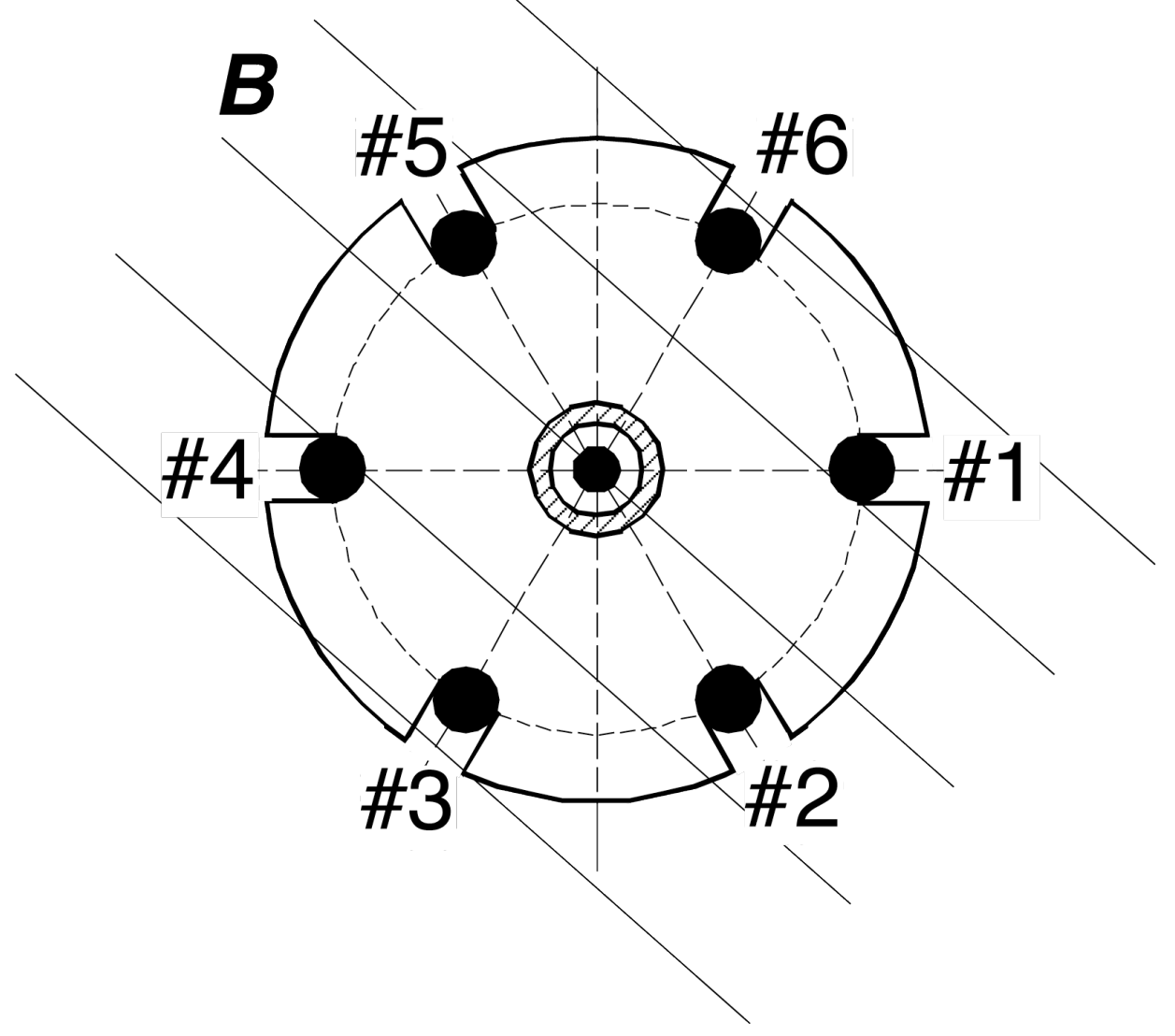


Figure 2

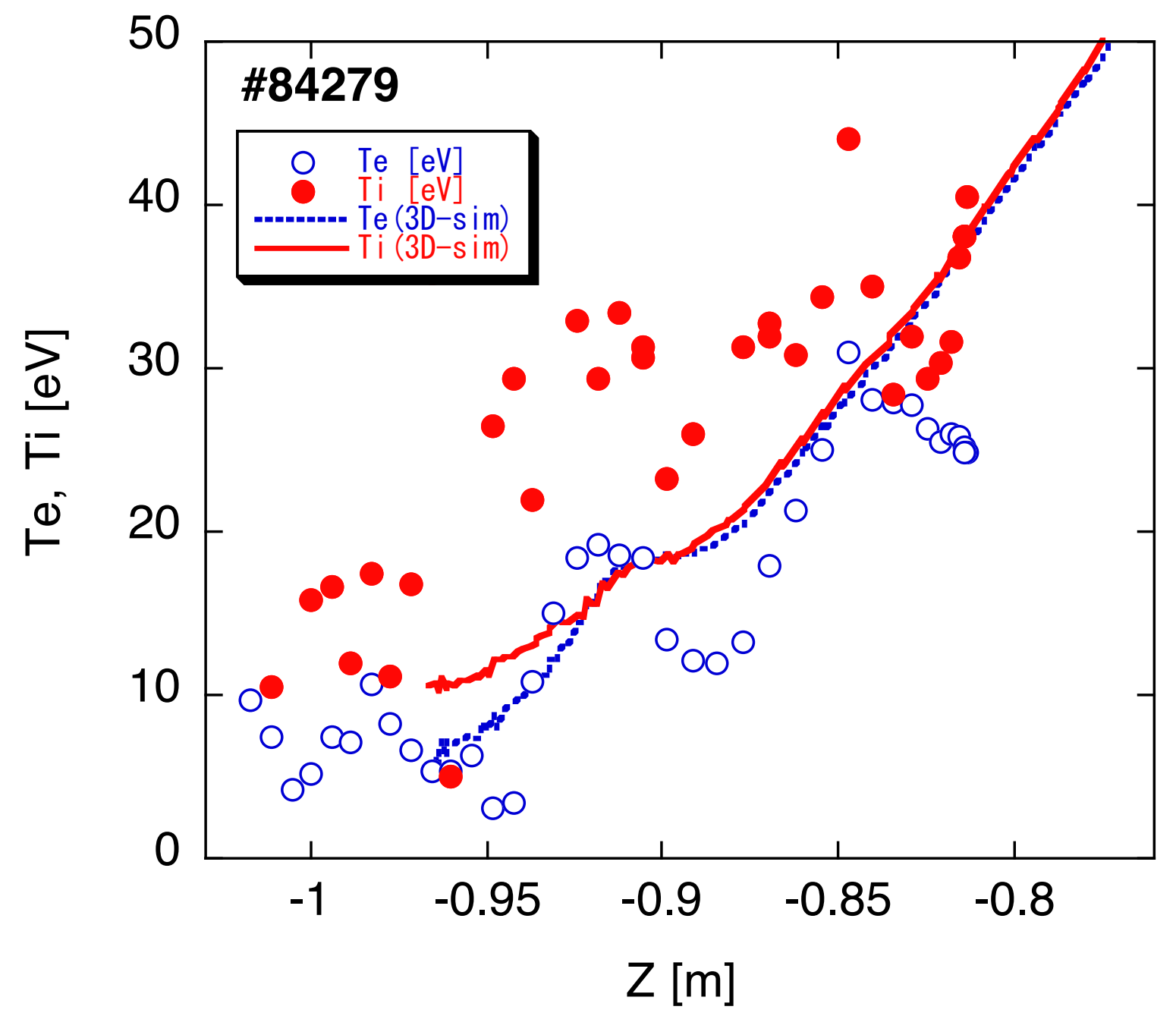


Figure 3

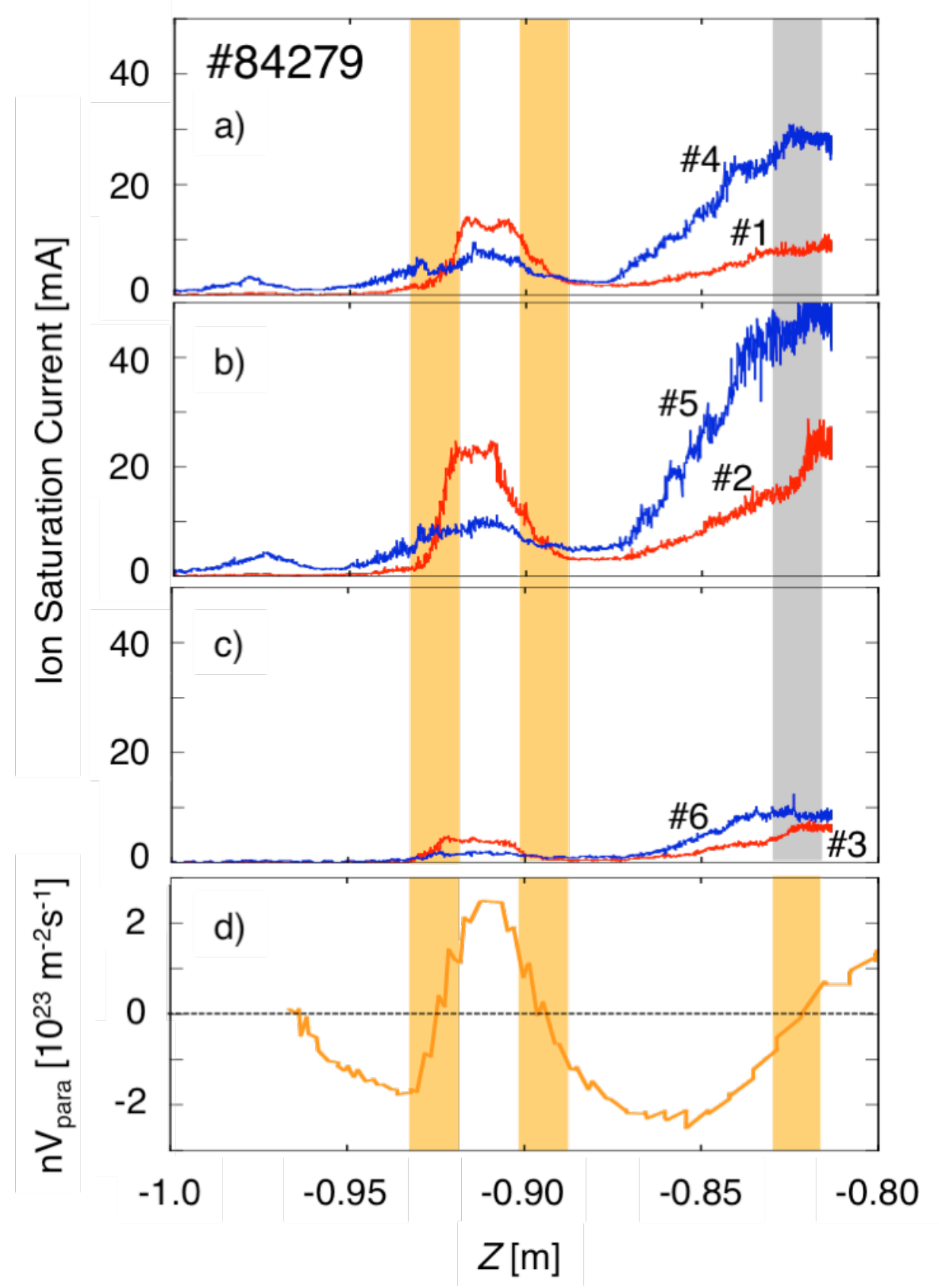

